\section{- OPEN ACCESS}

- Additional material is published online only. To view please visit the journal online (http://dx.doi.org/10.1136/ jclinpath-2013-201728).

${ }^{1}$ Affiliated Hospital of Academy of Military Medical Science, Beijing, China

${ }^{2}$ Innovation Center China, AstraZeneca Global R\&D, Shanghai, China ${ }^{3}$ Personalised Healthcare \& Biomarkers, AstraZeneca Global R\&D, Macclesfield, Cheshire, UK

\section{Correspondence to} Professor Xiaoging Liu, Affiliated Hospital of Academy of Military Medical Science, 8 Dong Da Street, Beijing 100071, China; liuxq@ medmail.com.cn

$\mathrm{XL}$ and $\mathrm{YL}$ contributed equally to the study.

Received 7 May 2013 Revised 26 June 2013 Accepted 27 June 2013 Published Online First 25 July 2013

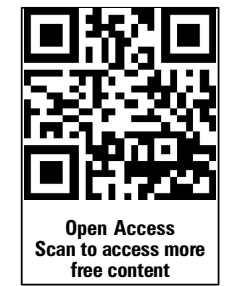

To cite: Liu X, Lu Y, Zhu G, et al. I Clin Pathol 2013;66:1065-1069.

\title{
The diagnostic accuracy of pleural effusion and plasma samples versus tumour tissue for detection of EGFR mutation in patients with advanced non-small cell lung cancer: comparison of methodologies
}

\author{
Xiaoqing Liu, ${ }_{1}$ Yachao Lu, ${ }^{2}$ Guanshan Zhu, ${ }^{2}$ Yao Lei, ${ }^{1}$ Li Zheng, ${ }^{2}$ Haifeng Qin, ${ }^{1}$ \\ Chuanhao Tang, ${ }^{1}$ Gillian Ellison, ${ }^{3}$ Rose McCormack, ${ }^{3}$ Qunsheng $\mathrm{Ji}^{2}$
}

\begin{abstract}
Aims To evaluate the suitability of malignant pleural effusion (MPE) and plasma as surrogate samples for epidermal growth factor receptor (EGFR) mutation detection, and compare three different detection methods.
\end{abstract}

Methods Matched tissue and plasma samples were collected from patients with advanced non-small cell lung cancer (NSCLC) (stage IIIB/IV adenocarcinoma/ adenosquamous carcinoma), with matched MPE samples collected from a subgroup. DNA was extracted from tissue, MPE cell block, MPE supernatant and plasma before mutation detection by amplification refractory mutation system (ARMS) (all samples), Sanger sequencing and mutant-specific immunohistochemistry (IHC) (tissue and MPE cell blocks only).

Results Sensitivity of MPE cell block, MPE supernatant and plasma versus tissue: $81.8 \%$ (9/11), 63.6\% (7/11) and $67.5 \%(27 / 40)$; specificity was $80.0 \%(8 / 10)$, $100 \%(10 / 10)$ and $100 \%(46 / 46)$, respectively. Sensitivity of Sanger sequencing versus ARMS: $81.8 \%$ (27/33) for tissue, 40\% (4/10) for MPE cell blocks; specificity was $100 \%$ (36/36 and 12/12) for both. Sensitivity of mutant-specific IHC versus ARMS: $54.8 \%$ (17/31) for tissue, $50.0 \%$ (6/12) for MPE cell blocks; specificity was $97.1 \%(34 / 35)$ and $100 \%$ (14/14), respectively.

Conclusions MPE and plasma are valid surrogates for NSCLC tumour EGFR mutation detection when tissue is not available. ARMS is most suitable for mutation detection in tissue and MPE cell blocks; however, mutant-specific IHC could be a complementary method when DNA-based molecular testing is unavailable.

\section{INTRODUCTION}

Targeted epidermal growth factor receptor (EGFR) tyrosine kinase inhibitors (TKIs), such as gefitinib and erlotinib, are approved for patients with EGFR mutation-positive advanced non-small cell lung cancer (aNSCLC), ${ }^{1}{ }^{2}$ and EGFR-activating mutations are an accepted molecular biomarker to predict EGFR TKI clinical efficacy.

Clinical application of EGFR mutation testing has progressed significantly, although sample availability remains a challenge. Surgery or biopsy tumour samples are preferred for optimal EGFR mutation detection, but are not always available. For example, $10-50 \%$ of patients with aNSCLC experience malignant pleural effusion (MPE), ${ }^{3-5}$ for whom pathological diagnosis may rely exclusively on finding cancer cells in the MPE. ${ }^{6}$ However, data detailing the sensitivity/specificity of testing MPE samples for EGFR mutations are limited. $^{7} 8$ Although currently restricted to the research setting, ${ }^{9-15}$ peripheral blood containing circulating-free DNA (cfDNA) from cancer cells ${ }^{7816}$ may be an alternative. ${ }^{9-16}$

EGFR mutation testing methodology presents another challenge due to the number of different methodologies available. ${ }^{8}{ }^{17}$ Among these, the widely available, novel mutation-detecting Sanger sequencing method has become established as the 'gold standard'. ${ }^{18}$ However, its limitations include low sensitivity, requirement for high-quality tumour samples, longer turn-around time and high reliance on staff experience. ${ }^{19} 20$ To overcome these disadvantages, targeted PCR-based methods (eg, amplification refractory mutation system (ARMS)) have been developed and are becoming widely accepted clinically, although they are unable to detect novel mutations. Alternatively, mutantspecific immunohistochemistry (IHC) has the potential to be more easily integrated in pathology laboratories, requires a small amount of material, eliminates the need for DNA and has a very rapid turn-around time, in addition to providing a quantitative assessment of mutation-positive cells, which other methods cannot do. To date, mutant-specific antibodies have been developed to detect the two most common EGFR mutations: an exon 19 deletion (E746-A750del) and the L858R mutation in exon $21,{ }^{21}$ but this method shows inconsistent sensitivity/specificity. ${ }^{22-29}$

We collected matched tissue, MPE and plasma samples from each patient to evaluate the potential of MPE and plasma as surrogate samples for EGFR mutation detection, and compared three different mutation detection technologies: ARMS, Sanger sequencing and mutant-specific IHC. This article focuses on sensitivity/specificity data.

\section{MATERIALS AND METHODS \\ Patients and samples}

Patients with advanced lung adenocarcinoma/adenosquamous carcinoma who provided informed consent at one centre in Beijing, China, from January 2008 to March 2012, were recruited. The study was approved by the hospital institutional ethics committee. Collection of tumour tissue sample and matched plasma was mandatory; collection of a matched pleural effusion sample was 
optional but encouraged. Further details relating to tissue, MPE and plasma sampling and handling, including DNA extraction and quality assurance, are provided in online supplementary appendix 1 .

\section{EGFR mutation detection}

Three methods were used to detect EGFR mutations in the different types of samples. ARMS was used for all four sample types: tumour tissue, MPE cell block, MPE supernatant and plasma. Sanger sequencing and mutant-specific IHC were used for tumour tissue and MPE cell block samples only. Based on previous evaluation and availability of appropriate instruments, the ADx-ARMS kit was used for this study rather than the Qiagen Scorpion ARMS kit. We defined a cut-off of $2 \%$ tumour cell content as a sample quality check according to the minimum requirement of ARMS technology (about 1\% analytical sensitivity). Samples below this threshold were rejected. Further details are provided in online supplementary appendix 1.

\section{RESULTS}

\section{Patient characteristics}

In total, 86/124 patients provided both adequate tissue samples ( $\geq 2 \%$ tumour cells) and sufficient plasma samples to be included in the mutation analyses (table 1 and see online supplementary appendix 1).

\section{EGFR mutation status detected by ARMS}

All samples included in the current analyses were evaluable using ARMS. EGFR mutation detection rates were 46.5\% (40/ $86)$ in tumour tissue samples, 48.1\% (13/27) in MPE cell block samples, $33.3 \%(9 / 27)$ in MPE supernatant samples and $31.4 \%$ $(27 / 86)$ in plasma samples.

Table 1 Clinicopathological characteristics of patients and samples $(n=86)$

\begin{tabular}{ll}
\hline Characteristic & Value \\
\hline Age, years & \\
Median & 55 \\
Range & $28-81$ \\
Sex, $\mathrm{n}(\%)$ & \\
Male & $56(65)$ \\
Female & $30(35)$ \\
Smoking history, $\mathrm{n}(\%)$ & \\
Never smoker & $39(45)$ \\
Former smoker & $10(12)$ \\
Current smoker & $37(43)$ \\
Histology, $\mathrm{n}$ (\%) & \\
Adenocarcinoma & $85(99)$ \\
Adenosquamous carcinoma & $1(1)$ \\
Disease stage, n (\%) & \\
IIIB & $4(5)$ \\
IV & $82(95)$ \\
Sample collection, n (\%) & $86(100)$ \\
Tumour tissue sample & $21(24)$ \\
Matched pleural effusion & $6(7)$ \\
Unmatched pleural effusion & 8600 \\
Matched plasma & \\
\hline & \\
\hline
\end{tabular}

EGFR mutation status detected by Sanger sequencing

A number of samples failed Sanger sequencing: 19.8\% (17/86) of tumour tissue samples and 18.5\% (5/27) of MPE cell block samples. EGFR mutation-positive rates were reported in successful analyses for $39.1 \%(27 / 69)$ of tumour tissue samples and $18.1 \%(4 / 22)$ of MPE cell block samples.

\section{EGFR mutation status detected by mutant-specific IHC}

Totally, 24.4\% (21/86) of tumour tissue samples and 3.7\% (1/ 27) of MPE cell block samples did not show total EGFR expression and were excluded from the mutation detection using IHC. Among the remaining total EGFR-expressed samples, EGFR mutation positive rates were $27.7 \%(18 / 65)$ in tumour tissue samples and 26.9\% (7/26) in MPE cell block samples.

\section{Comparison of different sample types using ARMS}

Tumour tissue versus MPE samples

Twenty-one patients provided adequate tumour tissue samples, matched adequate MPE cell block samples and matched MPE supernatant samples. Compared with tumour tissue samples, the sensitivity and specificity of MPE cell block samples for EGFR mutation detection were $81.8 \%$ and $80.0 \%$, respectively (table $2 \mathrm{~A}$ );

Table 2 Comparison of EGFR mutation status detected by ARMS in different sample types

\begin{tabular}{lccc}
\hline A. Tumour tissue versus MPE cell block & \\
\hline & \multicolumn{2}{c}{ Tumour tissue } & \\
\cline { 2 - 4 } MPE cell block & + & - & Total \\
\hline+ & 9 & 2 & 11 \\
- & 2 & 8 & 10 \\
Total & 11 & 10 & 21
\end{tabular}

NPV: $8 / 10$ (80.0\%); PPV: $9 / 11$ (81.8\%).

B. Tumour tissue versus MPE supernatant

\begin{tabular}{lcrr}
\hline & \multicolumn{2}{c}{ Tumour tissue } & \\
MPE supernatant & + & - & Tota \\
\hline+ & 7 & 0 & 7 \\
- & 4 & 10 & 14 \\
Total & 11 & 10 & 21
\end{tabular}

NPV: 10/14 (71.4\%); PPV: $7 / 7$ (100.0\%).

C. MPE cell block versus MPE supernatant

\begin{tabular}{lcrr}
\hline & \multicolumn{2}{c}{ MPE cell block } & \\
MPE supernatant & + & - & Total \\
\hline+ & 9 & 0 & 9 \\
- & 4 & 14 & 18 \\
Total & 13 & 14 & 27
\end{tabular}

NPV: $14 / 18(77.8 \%) ;$ PPV: $9 / 9(100.0 \%)$

D. Tumour tissue versus plasma

\begin{tabular}{lcrc}
\hline & \multicolumn{2}{c}{ Tumour tissue } & \\
Plasma & + & - & Total \\
\hline+ & 27 & 0 & 27 \\
- & 13 & 46 & 59 \\
Total & 40 & 46 & 86 \\
NPV: $46 / 59(79.0 \%) ;$ PPV: $27 / 27(100.0 \%)$. & &
\end{tabular}

ARMS, amplification refractory mutation system; EGFR, epidermal growth factor receptor; MPE, malignant pleural effusion; NPV, negative predictive value; PPV, positive predictive value. 
concordance was $81 \%(17 / 21)$. Compared with tumour tissue samples, the sensitivity and specificity of MPE supernatant samples were $63.6 \%(7 / 11)$ and $100 \%(10 / 10)$, respectively (table 2B); concordance was 81\% (17/21).

\section{MPE cell block versus MPE supernatant samples}

In the 27 patients who provided adequate MPE cell block samples and MPE supernatant samples, the EGFR mutation status concordance rate between MPE cell block and MPE supernatant samples was 85.2\% (23/27). Compared with MPE cell block samples, the sensitivity and specificity of MPE supernatant for EGFR mutation detection were 69.2\% (9/13) and $100 \%(14 / 14)$, respectively (table 2C).

\section{Tumour tissue versus plasma samples}

Compared with tumour tissue samples, the sensitivity and specificity of plasma for EGFR mutation detection were 67.5\% (27/ 40 ) and $100 \%(46 / 46)$, respectively (table 2D); concordance was $84.9 \%(73 / 86)$.

\section{Comparison of three different methods for EGFR mutation detection}

Although the success (pass) rate with ARMS was 100\% for tumour tissue samples and MPE cell block samples, the success rate with Sanger sequencing was 80.2\% (69/86) and 81.5\% (22/ $27)$, respectively. After total EGFR expression assessment by IHC, only $75.6 \%(65 / 86)$ of tumour tissue samples and $96.3 \%$ (26/27) of MPE cell block samples were judged adequate for mutant-specific IHC testing.

Sixty-nine tumour tissue samples were successfully tested by both ARMS and Sanger sequencing. Compared with ARMS, the sensitivity and specificity of Sanger sequencing for mutation detection were $81.8 \%(27 / 33)$ and $100 \%(36 / 36)$, respectively (table 3A); concordance was 91.3\% (63/69). Twenty-two MPE cell block samples were successfully tested by both ARMS and Sanger sequencing. Compared with ARMS, the sensitivity and specificity of Sanger sequencing for EGFR mutation detection were $40 \%(4 / 10)$ and $100 \%(12 / 12)$, respectively (table 3B); concordance was $72.7 \%(16 / 22)$.

Sixty-six tumour tissue samples were successfully tested by both ARMS and mutant-specific IHC. Compared with ARMS, the sensitivity and specificity of IHC for mutation detection were 54.8\% (17/31) and 97.1\% (34/35), respectively (table 3C); concordance was $77.3 \%$ (51/66). Among the IHC mutationpositive cases, the ratio of E19 del:L858R was $10: 7$, which was similar to the 19:13 ratio for ARMS mutation-positive cases. Twenty-six MPE cell block samples were successfully tested by both ARMS and IHC. Compared with ARMS, the sensitivity and specificity of IHC for mutation detection were $50 \%(6 / 12)$ and $100 \%(14 / 14)$, respectively (table $3 \mathrm{D})$; concordance was $76.9 \%(20 / 26)$.

\section{DISCUSSION}

Using the sensitive ARMS method, we detected EGFR mutations in $46.5 \%$ of tumour tissue samples, $48.1 \%$ of MPE cell block samples, $33.3 \%$ of MPE supernatant samples and $31.4 \%$ of plasma samples. Compared with tumour tissue, the sensitivity and specificity were $81.8 \%$ and $80.0 \%$ for MPE cell blocks and $63.6 \%$ and $100 \%$ for MPE supernatant, respectively. EGFR mutations can be detected in MPE from patients with NSCLC, with various mutation-positive rates, and EGFR mutation status between MPE and paired tissue could be different. ${ }^{30-34}$ However, to date, there have been limited reports of the sensitivity and specificity of MPE for EGFR mutation detection defined
Table 3 Comparison of EGFR mutation status detected by different methods in tumour tissue and MPE cell block samples

\begin{tabular}{lccc}
\hline A. ARMS versus Sanger sequencing for tumour tissue samples \\
\hline & \multicolumn{2}{c}{ ARMS } & \\
\cline { 2 - 4 } Sanger sequencing & + & - & Total \\
\hline+ & 27 & 0 & 27 \\
- & 6 & 36 & 42 \\
Total & 33 & 36 & 69
\end{tabular}

NPV: 36/42 (85.7\%); PPV: $27 / 27$ (100.0\%).

B. ARMS versus Sanger sequencing for MPE cell block samples

\begin{tabular}{lccc}
\hline & ARMS & & \\
Sanger sequencing & + & - & Tota \\
\hline+ & 4 & 0 & 4 \\
- & 6 & 12 & 18 \\
Total & 10 & 12 & 22
\end{tabular}

NPV: $12 / 18$ (66.7\%); PPV: 4/4 (100.0\%).

C. ARMS versus mutant-specific IHC for tumour tissue samples

\begin{tabular}{|c|c|c|c|}
\hline \multirow[b]{2}{*}{ IHC } & \multicolumn{3}{|c|}{ ARMS } \\
\hline & + & - & Tota \\
\hline+ & 17 & 1 & 18 \\
\hline- & 14 & 34 & 48 \\
\hline Total & 31 & 35 & 66 \\
\hline
\end{tabular}

D. ARMS versus mutant-specific IHC for MPE cell block samples

\begin{tabular}{lccc}
\hline \multicolumn{4}{c}{ ARMS } \\
IHC & + & - & Total \\
\hline+ & 6 & 0 & 6 \\
- & 6 & 14 & 20 \\
Total & 12 & 14 & 26 \\
NPV: $14 / 20(75.0 \%) ;$ PPV: $6 / 6(100.0 \%)$. & & \\
\hline ARMS, amplification refractory mutation system; EGFR, epidermal growth factor \\
receptor; IHC, immunohistochemistry; MPE, malignant pleural effusion; NPV, negative \\
predictive value; PPV, positive predictive value.
\end{tabular}

by comparison against matched tumour tissue. ${ }^{35}$ From our current study, the sensitivity of MPE cell blocks for EGFR mutation detection was $81.8 \%$, potentially high enough for clinical adoption if tissue is unavailable. In two cases, EGFR mutation was detected in the MPE cell block but not in the tumour tissue by ARMS. As the other techniques confirmed these two cases as EGFR mutation-positive, the EGFR mutation-positive rate was 52.4\% (11/21) for both tumour tissue and MPE, further strengthening the argument for the use of MPE cell block samples. We also showed that, although the specificity of the MPE supernatant was 100\% versus MPE cell block and tumour tissue, the sensitivity of MPE supernatant was $69.2 \%$ versus MPE cell block and $63.6 \%$ versus tumour tissue. Therefore, if an adequate MPE cell block or tumour sample is available, MPE supernatant is not recommended for mutation testing.

Plasma cfDNA is generally increased in patients with lung cancer, but with significant interpatient variability. ${ }^{36}$ Also, cfDNA tends to be fragmented, with DNA fragments $<200 \mathrm{bp},{ }^{37} 38$ and it can be contaminated with wild-type cfDNA. Therefore, reliable extraction of cfDNA extraction prior to sensitive methodology to amplify relatively short DNA fragments is essential. cfDNA EGFR mutation detection rates range from $36 \%$ to $92 \%$ versus paired NSCLC tumour 
tissue. $^{7}$ 9-11 $15 \quad 39$ Using ARMS, we found a 31.4\% EGFR mutation-positive rate in plasma cfDNA, with sensitivity of $67.5 \%$ and specificity of $100 \%$. The sensitivity from our study was similar to the $70 \%$ reported by Kuang et al ${ }^{11}$ who used ARMS and WAVE/Surveyor methods for plasma cfDNA EGFR mutation detection in patients with aNSCLC, but was much higher than the $43.1 \%$ reported by Goto et $\mathrm{al}^{7}$ who also used an older version of the ARMS method to detect EGFR mutations in serum cfDNA in a similar population. The use of a different ARMS method and serum samples may account for differences between our study and that of Goto et $a l^{7}$

The positive predictive value from our study of cfDNA was $100 \%$, consistent with Goto et $a l^{7}$ using ARMS, and that reported by Yung et $a l^{12}$ using digital PCR, suggesting that the positive mutation results detected by ARMS and/or digital PCR using cfDNA are highly predictive of a mutation-positive tumour sample. This is essential when considering the potential use of cfDNA, only when tumour tissue is not available. However, because the negative predictive value of cfDNA was only $78 \%$ in our study, it is not always possible to detect tumour EGFR mutations using this sample type and tumour tissue should be used if available. While the success (pass) rate of ARMS was 100\% for tumour tissue and MPE cell blocks, success rates of Sanger sequencing were $80.2 \%$ and $81.5 \%$, and success rates of mutantspecific IHC were $75.6 \%$ and $96.3 \%$, respectively.

Sanger sequencing failed to detect $\sim 20 \%$ of mutation-positive tumour samples (by ARMS) and 60\% of mutation-positive MPE cell block samples; $20 \%$ of samples also failed sequencing, demonstrating the inadequacy of Sanger methodology to detect EGFR mutations in some clinical samples. Sanger sequencing did not detect any novel mutation that would not have been detected using ARMS (which detects $>90 \%$ of all EGFR mutations). Analysis of the yield of amplifiable DNA and tumour content and their relationship with the performance of Sanger sequencing revealed that $95 \%$ of samples that failed DNA sequencing (9/17) had DNA $<0.4 \mathrm{ng} / \mu \mathrm{L}$. Among these, only $25 \%$ passed sequencing (3/12), whereas in samples with DNA $\geq 0.4 \mathrm{ng} / \mu \mathrm{L}, 89 \%$ passed (66/74). In clinical formalin-fixed, paraffin-embedded samples, low yield of amplifiable DNA is therefore a major factor leading to failure of EGFR mutation detection with Sanger sequencing. When we raised the tumour content cut-off from $1 \%$ to $20 \%$, the sensitivity of EGFR mutation detection with Sanger sequencing versus ARMS was 92.3\% (24/26); and specificity remained at 100\% (27/27), highlighting that when employing Sanger sequencing, samples must be of sufficient quality/quantity, with sufficient DNA for amplification.

Consistent with previous reports, our data showed that the mutant-specific IHC method was highly specific. ${ }^{22-29}$ However, it detected considerably fewer mutants than ARMS, and even fewer than Sanger sequencing for tumour tissue, but marginally more than Sanger sequencing for MPE cell block samples. Sensitivity of IHC in this study is considerably lower than in most previously reported studies. ${ }^{22-29}$ Possible explanations include: (1) the significant portion of archival tumour tissue samples, which could compromise EGFR protein detection and (2) variations in sample processing and IHC assay conditions. Nevertheless, considering the high specificity and wide availability of IHC in hospitals, this method should be encouraged if DNA-based molecular testing is not available.

Limitations of this study include the relatively small sample size, especially of MPE samples, and lack of clinical response data. Further investigations involving a greater number of samples with correlative clinical outcomes would also be a useful supplement.
In conclusion, MPE and plasma are valid surrogates for NSCLC tumour EGFR mutation detection when tissue is not available. ARMS is most suitable for mutation detection in tissue and MPE cell blocks; however, mutant-specific IHC could be a complementary method when DNA-based molecular testing is unavailable.

\section{Take-home messages}

- EGFR mutations in NSCLC predict treatment outcomes and guide patient selection for EGFR TKI therapy.

- Several established and emerging methods exist for the determination of EGFR mutations, most notably Sanger sequencing, ARMS and mutant-specific IHC.

- Determination of the sensitivity and specificity of these methods using paired tumour tissue and MPE or plasma samples revealed that MPE and plasma samples are valid surrogates for NSCLC tumour EGFR mutation detection when tissue is not available.

- ARMS is most suitable for mutation detection in tissue and MPE cell blocks; however, mutant-specific IHC could be a complementary method when DNA-based molecular testing is unavailable.

Acknowledgements We thank Changting Liu and Yanping $\mathrm{Xu}$ for their contribution in sample collection and preparation, Xiaolu Yin for her pathology support and Sunny Sun for her contribution to experimental data generation. We also thank Dr Yi Liu for participating in the initial study design discussion and help with sample collection. Editing assistance was provided by Sarah Lewis, from Complete Medical Communications, and funded by AstraZeneca.

Contributors All authors participated in the study design, collection, analysis and interpretation of data, in the writing of the manuscript and in the decision to submit the manuscript for publication. All authors reviewed the draft manuscript, and read and approved the final version for submission. $\mathrm{XL}$ and $\mathrm{YL}$ contributed equally to the study.

Funding This study was funded by AstraZeneca. Study design, EGFR mutation detection, data analysis, writing and decision to submit this manuscript for publication was supported by AstraZeneca.

Competing interests Guanshan Zhu, Yachao Lu, Li Zheng and Qunsheng Ji are employees of AstraZeneca. Gillian Ellison and Rose McCormack are employees of AstraZeneca and hold shares in AstraZeneca. Xiaoqing Liu, Yao Lei, Haifeng Qin and Chuanhao Tang have no conflicts of interest to disclose.

Ethics approval Ethics approval for this study was given by the Affiliated Hospital of Academy of Military Medical Science Institutional Ethics Committee.

Provenance and peer review Not commissioned; externally peer reviewed.

Open Access This is an Open Access article distributed in accordance with the Creative Commons Attribution Non Commercial (CC BY-NC 3.0) license, which permits others to distribute, remix, adapt, build upon this work non-commercially, and license their derivative works on different terms, provided the original work is properly cited and the use is non-commercial. See: http://creativecommons.org/ licenses/by-nc/3.0/

\section{REFERENCES}

1 Peters S, Adjei AA, Gridelli C, et al. Metastatic non-small-cell lung cancer (NSCLC): ESMO Clinical Practice Guidelines for diagnosis, treatment and follow-up. Ann Oncol 2012;23(Suppl 7):vii56-64.

2 Azzoli CG, Temin S, Aliff T, et al. 2011 Focused Update of 2009 American Society of Clinical Oncology Clinical Practice Guideline Update on Chemotherapy for Stage IV Non-Small-Cell Lung Cancer. J Clin Oncol 2011;29:3825-31.

3 Zhang $X$, Zhao $Y$, Wang $M$, et al. Detection and comparison of epidermal growth factor receptor mutations in cells and fluid of malignant pleural effusion in non-small cell lung cancer. Lung Cancer 2008;60:175-82.

4 Fenton KN, Richardson JD. Diagnosis and management of malignant pleural effusions. Am J Surg 1995;170:69-74. 
5 Sekine I, Sumi M, Saijo N. Local control of regional and metastatic lesions and indication for systemic chemotherapy in patients with non-small cell lung cancer. Oncologist 2008:13(Suppl 1):21-7.

6 Antony VB, Loddenkemper $\mathrm{R}$, Astoul $\mathrm{P}$, et al. Management of malignant pleural effusions. Eur Respir J 2001;18:402-19.

7 Goto $\mathrm{K}$, Ichinose $\mathrm{Y}$, Ohe $\mathrm{Y}$, et al. Epidermal growth factor receptor mutation status in circulating free DNA in serum: from IPASS, a phase III study of gefitinib or carboplatin/paclitaxel in non-small cell lung cancer. J Thorac Oncol 2012;7:115-21.

8 Goto K, Satouchi M, Ishii G, et al. An evaluation study of EGFR mutation tests utilized for non-small-cell lung cancer in the diagnostic setting. Ann Oncol 2012;23:2914-19.

9 Bai H, Mao L, Wang HS, et al. Epidermal growth factor receptor mutations in plasma DNA samples predict tumor response in Chinese patients with stages IIIB to IV non-small-cell lung cancer. J Clin Oncol 2009;27:2653-9.

10 Brevet M, Johnson ML, Azzoli CG, et al. Detection of EGFR mutations in plasma DNA from lung cancer patients by mass spectrometry genotyping is predictive of tumor EGFR status and response to EGFR inhibitors. Lung Cancer 2011;73:96-102.

11 Kuang Y, Rogers A, Yeap BY, et al. Noninvasive detection of EGFR T790M in gefitinib or erlotinib resistant non-small cell lung cancer. Clin Cancer Res 2009;15:2630-6.

12 Yung TK, Chan KC, Mok TS, et al. Single-molecule detection of epidermal growth factor receptor mutations in plasma by microfluidics digital PCR in non-small cell lung cancer patients. Clin Cancer Res 2009;15:2076-84.

13 Mack PC, Holland WS, Burich RA, et al. EGFR mutations detected in plasma are associated with patient outcomes in erlotinib plus docetaxel-treated non-small cell lung cancer. J Thorac Oncol 2009:4:1466-72.

14 Nakamura T, Sueoka-Aragane N, Iwanaga K, et al. A noninvasive system for monitoring resistance to epidermal growth factor receptor tyrosine kinase inhibitors with plasma DNA. J Thorac Oncol 2011;6:1639-48

15 Zhao X, Han RB, Zhao J, et al. Comparison of Epidermal Growth Factor Receptor Mutation Statuses in Tissue and Plasma in Stage I-IV Non-Small Cell Lung Cancer Patients. Respiration 2013;85:119-25.

16 Schwarzenbach H, Hoon DS, Pantel K. Cell-free nucleic acids as biomarkers in cancer patients. Nat Rev Cancer 2011;11:426-37.

17 Angulo B, Conde E, Suarez-Gauthier A, et al. A comparison of EGFR mutation testing methods in lung carcinoma: direct sequencing, real-time PCR and immunohistochemistry. PLoS One 2012;7:e43842.

18 Bakker E. Is the DNA sequence the gold standard in genetic testing? Quality of molecular genetic tests assessed. Clin Chem 2006;52:557-8.

19 Ogino S, Kawasaki T, Brahmandam M, et al. Sensitive sequencing method for KRAS mutation detection by Pyrosequencing. J Mol Diagn 2005;7:413-21.

20 Li J, Wang L, Mamon $\mathrm{H}$, et al. Replacing PCR with COLD-PCR enriches variant DNA sequences and redefines the sensitivity of genetic testing. Nat Med 2008;14:579-84.

$21 \mathrm{Yu}$ J, Kane S, Wu J, et al. Mutation-specific antibodies for the detection of EGFR mutations in non-small-cell lung cancer. Clin Cancer Res 2009;15:3023-8.

22 Simonetti S, Molina MA, Queralt C, et al. Detection of EGFR mutations with mutation-specific antibodies in stage IV non-small-cell lung cancer. J Trans/ Med 2010;8:135.

23 Kozu Y, Tsuta K, Kohno T, et al. The usefulness of mutation-specific antibodies in detecting epidermal growth factor receptor mutations and in predicting response to tyrosine kinase inhibitor therapy in lung adenocarcinoma. Lung Cancer 2011;73:45-50

24 Ilie Ml, Hofman V, Bonnetaud C, et al. Usefulness of tissue microarrays for assessment of protein expression, gene copy number and mutational status of EGFR in lung adenocarcinoma. Virchows Arch 2010;457:483-95.

25 Kato Y, Peled N, Wynes MW, et al. Novel epidermal growth factor receptor mutation-specific antibodies for non-small cell lung cancer: immunohistochemistry as a possible screening method for epidermal growth factor receptor mutations. J Thorac Oncol 2010;5:1551-8.

26 Brevet $\mathrm{M}$, Arcila M, Ladanyi M. Assessment of EGFR mutation status in lung adenocarcinoma by immunohistochemistry using antibodies specific to the two major forms of mutant EGFR. J Mol Diagn 2010;12:169-76.

27 Tsai T-H, Wu S-G, Chang Y-L, et al. Effusion immunocytochemistry as an alternative approach for the selection of first-line targeted therapy in advanced lung adenocarcinoma. J Thorac Oncol 2012;7:993-1000.

28 Wu SG, Chang YL, Lin JW, et al. Including total EGFR staining in scoring improves EGFR mutations detection by mutation-specific antibodies and EGFR TKIs response prediction. PloS One 2011;6:e23303.

29 Hasanovic A, Ang D, Moreira AL, et al. Use of mutation specific antibodies to detect EGFR status in small biopsy and cytology specimens of lung adenocarcinoma. Lung Cancer 2012;77:299-305.

30 Kimura $H$, Fujiwara $Y$, Sone $T$, et al. High sensitivity detection of epidermal growth factor receptor mutations in the pleural effusion of non-small cell lung cancer patients. Cancer Sci 2006;97:642-8

31 Kimura $\mathrm{H}$, Fuijwara $\mathrm{Y}$, Sone $\mathrm{T}$, et al. EGFR mutation status in tumour-derived DNA from pleural effusion fluid is a practical basis for predicting the response to gefitinib. Br J Cancer 2006;95:1390-5.

32 Soh J, Toyooka S, Aoe K, et al. Usefulness of EGFR mutation screening in pleural fluid to predict the clinical outcome of gefitinib treated patients with lung cancer. Int J Cancer 2006;119:2353-8.

33 Wu SG, Gow CH, Yu CJ, et al. Frequent epidermal growth factor receptor gene mutations in malignant pleural effusion of lung adenocarcinoma. Eur Respir J 2008;32:924-30.

34 Buttitta F, Felicioni L, Del GM, et al. Effective assessment of egfr mutation status in bronchoalveolar lavage and pleural fluids by next-generation sequencing. Clin Cancer Res 2013;19:691-8.

35 Han HS, Lim SN, An JY, et al. Detection of EGFR mutation status in lung adenocarcinoma specimens with different proportions of tumor cells using two methods of differential sensitivity. J Thorac Oncol 2012;7:355-64.

36 Jahr $\mathrm{S}$, Hentze $\mathrm{H}$, Englisch $\mathrm{S}$, et al. DNA fragments in the blood plasma of cancer patients: quantitations and evidence for their origin from apoptotic and necrotic cells. Cancer Res 2001;61:1659-65.

37 Suzuki N, Kamataki A, Yamaki J, et al. Characterization of circulating DNA in healthy human plasma. Clin Chim Acta 2008;387:55-8.

38 Yuan $\mathrm{H}$, Zhu ZZ, Lu Y, et al. A modified extraction method of circulating free DNA for epidermal growth factor receptor mutation analysis. Yonsei Med J 2012;53:132-7.

39 Hirsch FR, Bunn PA Jr. EGFR testing in lung cancer is ready for prime time. Lancet Oncol 2009;10:432-3. 